Vietnam Journal of Mechanics, NCST of Vietnam Vol. 25, 2003, No 1 (9-18)

\title{
ON THE ELASTO-PLASTIC STABILITY PROBLEM OF SHELLS OF REVOLUTION
}

\author{
DAO HUY BICH \\ Hanoi National University
}

\begin{abstract}
Summary. This paper deals with the elasto-plastic stability problems of shells of revolution subjected to complex loading process. The governing equations were derived and were solved by using the Bubnov-Galerkin method and the loading parameter method. Some examples were considered.
\end{abstract}

\section{Introduction}

Numerous solutions of the elasto-plastic stability problems of rectangular plates and circular cylindrical shells have been published in the literature by using different theories of plasticity. The critical loads acting on these structures were determined and the influence on which of the complex loading process was considered.

Many structural shell configurations are shells of revolution, the elastic stability problems of which were investigated widely, but for elasto-plastic problems there was a few only results, especially when considering the complex loading process acting on these structures $[5,6,8,10]$.

In this paper by using the theory of elasto-plastic processes and the adjacentequilibrium criterion we derive the governing equations of the elasto-plastic stability problem of shells of revolution. Here we restrict ourselves, the applied load is axisymmetric and the linear bending equations are used for the prebuckling deformation. The Bubnov-Galerkin method and the loading parameter method are applied in solving problem.

For illustration we consider the axisymmetric buckling of a circular plate subjected to uniform compressive loading and a shallow spherical cap subjected to uniform external pressure. From the obtained results we can get again the results of Timoshenko and Hutchinson for elastic shells, this fact provides the reliability of the obtained results.

\section{Prebuckling state of a shell of revolution}

Let us consider a shell of revolution, the middle surface of which may be form by rotation of a plane curve about an axis in the plane of the curve. Planes normal to axis of revolution intersect the surface in curves called parallels and planes that contain the axis intersect the surface in curves called meridians. Points on the surface 
may be referred to coordinates $\varphi, \theta$, where $\varphi$ denotes the angle between the axis of revolution and a normal to the surface and $\theta$ is a circumferential coordinate. The principal radii of curvature of the surface in the $\varphi$ and $\theta$ directions may be denoted by $R_{2}$ and $R_{1}$ respectively. It is convenient to introduce an additional variable $r$ defined by the relation

$$
r=R_{1} \sin \varphi, \quad \frac{d r}{d \varphi}=R_{2} \cos \varphi
$$

If the applied load is axisymmetric, the deformation also is axisymmetric prior to loss of stability. In the prebuckling state the membrane forces $N_{\varphi}^{0}, N_{\theta}^{0}, N_{\varphi \theta}^{0}$ are functions of $\varphi$ alone, they satisfy the corresponding linear membrane equations [3]

$$
\begin{aligned}
\frac{d}{d \varphi}\left(r N_{\varphi}^{0}\right)-R_{2} N_{\theta}^{0} \cos \varphi & =-r R_{2} p_{\varphi}, \\
\frac{d}{d \varphi}\left(r N_{\varphi \theta}^{0}\right)+R_{2} N_{\varphi \theta}^{0} \cos \varphi & =-r R_{2} p_{\theta} \\
r N_{\varphi}^{0}+R_{2} N_{\theta}^{0} \sin \varphi & =r R_{2} p_{z} .
\end{aligned}
$$

where $N_{\varphi}^{0}=h \sigma_{\varphi}^{0}, N_{\theta}^{0}=h \sigma_{\theta}^{0}, N_{\varphi \theta}^{0}=h \sigma_{\varphi \theta}^{0}$.

Equations are seen to be statically determinate, so that solutions can be obtained without use of constitutive and kinematic relations. If the shell is not subjected to torsional loading, $N_{\varphi \theta}^{0}=0$ and the second of equations is discarded.

\section{Stability equations}

The linear stability equation may be obtained by application of the adjacentequilibrium criterion. For this purpose we put

$$
\begin{gathered}
u=u_{0}+\delta u \\
v=v_{0}+\delta v \\
w=w_{0}+\delta w
\end{gathered}
$$

where $\left(u_{0} ; v_{0}, w_{0}\right)$ represent the equilibrium configuration whose stability is under consideration, $(u, v, w)$ is an adjacent equilibrium configuration corresponding to the same value of applied load as configuration $\left(u_{0}, v_{0}, w_{0}\right)$ and $(\delta u, \delta v, \delta w)$ is an arbitrary small incremental displacement. Furthermore

$$
\begin{aligned}
N_{\varphi} & =N_{\varphi}^{0}+\delta N_{\varphi}, & M_{\varphi} & =\delta M_{\varphi} \\
N_{\theta} & =N_{\theta}^{0}+\delta N_{\theta}, & M_{\theta} & =\delta M_{\theta} \\
N_{\varphi \theta} & =N_{\varphi \theta}^{0}+\delta N_{\varphi \theta}, & M_{\varphi \theta} & =\delta M_{\varphi \theta},
\end{aligned}
$$

where $\delta N_{\varphi}, \delta N_{\theta}, \delta N_{\varphi \theta}$ and $\delta M_{\varphi}, \delta M_{\theta}, \delta M_{\varphi \theta}$ are generalized force and moment increments corresponding to $(\delta u, \delta v, \delta w)$. Although the prebuckling state is axisymmetric, but the post-buckling is more general, so that the stability equations of shells 
of revolution may be obtained as follows [1]:

$$
\begin{aligned}
& \frac{\partial}{\partial \varphi}\left(r \delta N_{\varphi}\right)+R_{2} \frac{\partial}{\partial \theta}\left(\delta N_{\varphi \theta}\right)-R_{2} \delta N_{\theta} \cos \varphi=0, \\
& \frac{\partial}{\partial \varphi}\left(r \delta N_{\varphi \theta}\right)+R_{2} \frac{\partial}{\partial \theta}\left(\delta N_{\theta}\right)+R_{2} \delta N_{\varphi \theta} \cos \varphi=0, \\
& \frac{\partial}{\partial \varphi}\left[\frac{1}{R_{2}} \frac{\partial}{\partial \varphi}\left(r \delta M_{\varphi}\right)\right]+2\left(\frac{\partial^{2} \delta M_{\varphi \theta}}{\partial \theta^{2}}+\frac{R_{2}}{r} \frac{\partial \delta M_{\varphi \theta}}{\partial \theta} \cos \varphi\right)+ \\
& +\frac{R_{2}}{r} \frac{\partial^{2} \delta M_{\theta}}{\partial \theta^{2}}-\frac{\partial}{\partial \varphi}\left(\delta M_{\theta} \cos \varphi\right)-\left(r \delta N_{\varphi}+R_{2} \delta N_{\theta} \sin \varphi\right)- \\
& -\left[\frac{\partial}{\partial \varphi}\left(N_{\varphi}^{0} \delta \beta_{\varphi}+r N_{\varphi \theta}^{0} \delta \beta_{\theta}\right)+\frac{\partial}{\partial \theta}\left(R_{2} N_{\theta}^{0} \delta \beta_{\theta}+R_{2} N_{\varphi \theta}^{0} \delta \beta_{\varphi}\right)\right]=0,
\end{aligned}
$$

where

$$
\delta \beta_{\varphi}=\frac{1}{R_{2}} \frac{\partial \delta w}{\partial \varphi}, \quad \delta \beta_{\theta}=\frac{1}{r} \frac{\partial \delta w}{\partial \theta},
$$

$N_{\varphi}^{0}, N_{\theta}^{0}, N_{\varphi \theta}^{0}$ are determined from equations (2.2) of the prebuckling state.

According to the theory of elasto-plastic deformation processes the force and moment intensities are related to the internal stresses and deformation by the equations [4]

$$
\begin{aligned}
\delta N_{\varphi} & =h\left[\frac{2 N}{3}\left(2 \delta \varepsilon_{\varphi}^{*}+\delta \varepsilon_{\theta}^{*}\right)+\left(\phi^{\prime}-N\right) \frac{\sigma_{\varphi}^{0} \delta \varepsilon_{\varphi}^{*}+\sigma_{\theta}^{0} \delta \varepsilon_{\theta}^{*}+2 \sigma_{\varphi \theta}^{0} \delta \varepsilon_{\varphi \theta}^{*}}{\sigma_{u}^{0}} \sigma_{\varphi}^{0}\right] \\
\delta N_{\theta} & =h\left[\frac{2 N}{3}\left(2 \delta \varepsilon_{\theta}^{*}+\delta \varepsilon_{\varphi}^{*}\right)+\left(\phi^{\prime}-N\right) \frac{\sigma_{\varphi}^{0} \delta \varepsilon_{\varphi}^{*}+\sigma_{\theta}^{0} \delta \varepsilon_{\theta}^{*}+2 \sigma_{\varphi \theta}^{0} \delta \varepsilon_{\varphi \theta}^{*}}{\sigma_{u}^{02}} \sigma_{\theta}^{0}\right], \\
\delta N_{\varphi \theta} & =h\left[\frac{2 N}{3} \delta \varepsilon_{\varphi \theta}^{*}+\left(\phi^{\prime}-N\right) \frac{\sigma_{\varphi}^{0} \delta \varepsilon_{\varphi}^{*}+\sigma_{\theta}^{0} \delta \varepsilon_{\theta}^{*}+2 \sigma_{\varphi \theta}^{0} \delta \varepsilon_{\varphi \theta}^{*}}{\sigma_{u}^{0^{2}}} \sigma_{\varphi \theta}^{0}\right]
\end{aligned}
$$

and

$$
\begin{aligned}
\delta M_{\varphi} & =-\frac{h^{3}}{12}\left[\frac{2 N}{3}\left(2 \delta \chi_{\varphi}+\delta \chi_{\theta}\right)+\left(\phi^{\prime}-N\right) \frac{\sigma_{\varphi}^{0} \delta \chi_{\varphi}+\sigma_{\theta}^{0} \delta \chi_{\theta}+2 \sigma_{\varphi \theta}^{0} \delta \chi_{\varphi \theta}}{\sigma_{u}^{02}} \sigma_{\varphi}^{0}\right], \\
\delta M_{\theta} & =-\frac{h^{3}}{12}\left[\frac{2 N}{3}\left(2 \delta \chi_{\theta}+\delta \chi_{\varphi}\right)+\left(\phi^{\prime}-N\right) \frac{\sigma_{\varphi}^{0} \delta \chi_{\varphi}+\sigma_{\theta}^{0} \delta \chi_{\theta}+2 \sigma_{\varphi \theta}^{0} \delta \chi_{\varphi \theta}}{\sigma_{u}^{02}} \sigma_{\theta}^{0}\right], \\
\delta M_{\varphi \theta} & =-\frac{h^{3}}{12}\left[\frac{2 N}{3} \delta \chi_{\varphi \theta}+\left(\phi^{\prime}-N\right) \frac{\sigma_{\varphi}^{0} \delta \chi_{\varphi}+\sigma_{\theta}^{0} \delta \chi_{\theta}+2 \sigma_{\varphi \theta}^{0} \delta \chi_{\varphi \theta}}{\sigma_{u}^{02}} \sigma_{\varphi \theta}^{0}\right],
\end{aligned}
$$

where

$$
\begin{aligned}
N & =\frac{\sigma_{u}^{0}}{s}, \quad \phi^{\prime}=\frac{d \phi(s)}{d s} \\
\delta s & =\frac{2}{\sqrt{3}}\left(\delta \varepsilon_{\varphi}^{* 2}+\delta \varepsilon_{\theta}^{* 2}+\delta \varepsilon_{\varphi}^{*} \delta \varepsilon_{\theta}^{*}+\delta \varepsilon_{\varphi \theta}^{*}\right)^{1 / 2} \\
\sigma_{u}^{02} & =\sigma_{\varphi}^{02}+\sigma_{\theta}^{02}-\sigma_{\varphi}^{0} \sigma_{\theta}^{0}+3 \sigma_{\varphi \theta}^{02}
\end{aligned}
$$


and the linear middle surface kinematic relations are of the form

$$
\begin{aligned}
\delta \varepsilon_{\varphi}^{*} & =\frac{1}{R_{2}}\left(\frac{\partial \delta v}{\partial \varphi}-\delta w\right), \quad \delta \varepsilon_{\theta}^{*}=\frac{1}{r}\left(\frac{\partial \delta u}{\partial \theta}+\delta v \cos \varphi-\delta w \sin \varphi\right) \\
\delta \varepsilon_{\varphi \theta}^{*} & =\frac{1}{2}\left[\frac{r}{R_{2}} \frac{\partial}{\partial \varphi}\left(\frac{\delta u}{r}\right)+\frac{1}{r} \frac{\partial \delta v}{\partial \theta}\right], \quad \delta \chi_{\varphi}=\frac{1}{R_{2}} \frac{\partial \delta \beta_{\varphi}}{\partial \varphi}=\frac{1}{R_{2}} \frac{\partial}{\partial \varphi}\left(\frac{1}{R_{2}} \frac{\partial \delta w}{\partial \varphi}\right) \\
\delta \chi_{\theta} & =\frac{1}{r}\left(\frac{\partial \delta \beta_{\theta}}{\partial \theta}+\delta \beta_{\varphi} \cos \varphi\right)=\frac{1}{r}\left[\frac{\partial}{\partial \theta}\left(\frac{1}{r} \frac{\partial \delta w}{\partial \theta}\right)+\frac{1}{R_{2}} \frac{\partial \delta w}{\partial \varphi} \cos \varphi\right] \\
\delta \chi_{\varphi \theta} & =\frac{1}{2}\left[\frac{r}{R_{2}} \frac{\partial}{\partial \varphi}\left(\frac{\delta \beta_{\theta}}{r}\right)+\frac{1}{r} \frac{\partial \delta \beta_{\varphi}}{\partial \theta}\right]=\frac{1}{2}\left[\frac{r}{R_{2}} \frac{\partial}{\partial \varphi}\left(\frac{1}{r^{2}} \frac{\partial \delta w}{\partial \theta}\right)+\frac{1}{r} \frac{\partial}{\partial \theta}\left(\frac{1}{r} \frac{\partial \delta w}{\partial \varphi}\right)\right]
\end{aligned}
$$

The obtained equations $(3.1) \div(3.4)$ form a closed system of homogeneous equations of the elasto-plastic stability problem of shell of revolution. Combining boundary conditions to this system we can get the solution of the problem by using the Bubnov-Galerkin method and the loading parameter method. For illustration, solutions of these equations are discussed in consideration of circular plates and spherical caps.

\section{Circular plates}

The middle plane of a circular plate may be defined by polar coordinates $r$ and $\theta$. in specialization of the shell of revolution equations for the plate, $R_{1}$ and $R_{2}$ go to infinity, the angle $\varphi$ goes to zero, $\sin \varphi=0, \cos \varphi=1$ and $\lim _{R_{2} \rightarrow \infty} R_{2} d \varphi=d r$.

The equation (3.1) become

$$
\begin{aligned}
& \frac{\partial}{\partial r}\left(r \delta N_{r}\right)+\frac{\partial \delta N_{r \theta}}{\partial \theta}-\delta N_{\theta}=0, \\
& \frac{\partial}{\partial r}\left(r \delta N_{r \theta}\right)+\frac{\partial \delta N_{\theta}}{\partial \theta}+\delta N_{r \theta}=0, \\
& \frac{\partial^{2}}{\partial r^{2}}\left(r \delta M_{r}\right)+2\left(\frac{\partial^{2} \delta M_{r \theta}}{\partial r \partial \theta}+\frac{1}{r} \frac{\partial \delta M_{r \theta}}{\partial \theta}\right)+\frac{1}{r} \frac{\partial^{2} \delta M_{\theta}}{\partial \theta^{2}}-\frac{\partial \delta M_{\theta}}{\partial r}- \\
& -\left[\frac{\partial}{\partial r}\left(r N_{r}^{0} \delta \beta_{r}+r N_{r \theta}^{0} \delta \beta_{\theta}\right)+\frac{\partial}{\partial \theta}\left(N_{r \theta}^{0} \delta \beta_{r}+N_{\theta}^{0} \delta \beta_{\theta}\right)\right]=0
\end{aligned}
$$

where the subscript $\varphi$ has been replaced by $r$.

The equations (3.2), (3.3) are the same, but replacing subscript $\varphi$ by $r$. The relations (3.4) now are of the form

$$
\begin{aligned}
& \delta \varepsilon_{r}^{*}=\frac{\partial \delta v}{\partial r}, \quad \delta \varepsilon_{\theta}^{*}=\frac{1}{r}\left(\frac{\partial \delta u}{\partial \theta}+\delta v\right), \quad \delta \varepsilon_{r \theta}^{*}=\frac{1}{2}\left[r \frac{\partial}{\partial r}\left(\frac{\delta u}{r}\right)+\frac{1}{r} \frac{\partial \delta v}{\partial \theta}\right] \\
& \delta \chi_{r}=\frac{\partial \delta \beta_{r}}{\partial r}, \quad \delta \chi_{\theta}=\frac{1}{r}\left(\frac{\partial \delta \beta_{\theta}}{\partial \theta}+\delta \beta_{r}\right), \quad \delta \chi_{r \theta}=\frac{1}{2}\left[r \frac{\partial}{\partial r}\left(\frac{\delta \beta_{\theta}}{r}\right)+\frac{1}{r} \frac{\partial \delta \beta_{r}}{\partial \theta}\right] .
\end{aligned}
$$


For example we consider the axisymmetric buckling of a plate subjected to uniform compressive loading $P=p h$. Prebuckling state occurs in the plate

$$
\begin{aligned}
& \sigma_{r}^{0}=\sigma_{\theta}^{0}=-p, \quad N_{r}^{0}=N_{\theta}^{0}=-P, \quad N_{r \theta}^{0}=0 \\
& \sigma_{u}^{0}=\left|\sigma_{r}^{0}\right|=p, \quad \frac{d s}{d t}=2\left|\dot{\varepsilon}_{r}\right|, \quad \dot{\varepsilon}_{r}=-\frac{\dot{p}}{2 \phi^{\prime}(s)} \quad \text { (see [4]) }
\end{aligned}
$$

thus $\frac{d s}{d t}=\frac{\dot{p}}{\phi^{\prime}(s)}$ or $\phi^{\prime}(s) d s=d p$, i.e, $p=\phi(s)=\sigma_{u}^{0}$.

Because of axisymmetric buckling the determined quantities do nod depend on variable $\theta$ and $\delta \beta_{\theta}=0$. The third equation of (4.1) specializes to the expression

$$
\frac{d^{2}}{d r^{2}}\left(r \delta M_{r}\right)-\frac{d}{d r}\left(\delta M_{\theta}\right)+\frac{d}{d r}\left(r p h \delta \beta_{r}\right)=0
$$

and the relations $(3.3),(4.2)$ lead to the following

$$
\begin{aligned}
\delta \chi_{r} & =\frac{d \delta \beta_{r}}{d r}, \quad \delta \chi_{\theta}=\frac{\delta \beta_{r}}{r} \\
\delta M_{r} & =-\frac{h^{3}}{12}\left[\frac{2}{3} N\left(2 \frac{d \delta \beta_{r}}{d r}+\frac{\delta \beta_{r}}{r}\right)+\left(\phi^{\prime}-N\right)\left(\frac{d \delta \beta_{r}}{d r}+\frac{\delta \beta_{r}}{r}\right)\right] \\
\delta M_{\theta} & =-\frac{h^{3}}{12}\left[\frac{2}{3} N\left(2 \frac{\delta \beta_{r}}{r}+\frac{d \delta \beta_{r}}{d r}\right)+\left(\phi^{\prime}-N\right)\left(\frac{d \delta \beta_{r}}{d r}+\frac{\delta \beta_{r}}{r}\right)\right] .
\end{aligned}
$$

Integration of the equation (4.3) gives

$$
\frac{d}{d r}\left(r \delta M_{r}\right)-\delta M_{\theta}+r p h \delta \beta_{r}=C
$$

but $\delta M_{r} \equiv \delta M_{\theta} \equiv 0$ for $p=0$, then $C=0$. Substituting the expression of $\delta M_{r}$, $\delta M_{\theta}$ into the equation (4.5) we have

$$
r^{2} \frac{d^{2} \delta \beta_{r}}{d r^{2}}+r \frac{d \delta \beta_{r}}{d r}-\left(1-\frac{12 p}{\left(\phi^{\prime}+\frac{N}{3}\right) h^{2}} r^{2}\right) \delta \beta_{r}=0 .
$$

Let $\alpha^{2}=\frac{12 p}{\left(\phi^{\prime}+\frac{N}{3}\right) h^{2}}$, the general solution of $(4.6)$ is

$$
\beta_{r}=C_{1} J_{1}(\alpha r)+C_{2} Y_{1}(\alpha r)
$$

$J_{1}, Y_{1}$ are Bessel functions of first order of the first and second kinds respectively. But $\delta \beta_{r}=0$ at $r=0, Y_{1}(0)=\infty$, therefore $C_{2}=0$ and $\delta \beta_{r}=C_{1} J_{1}(\alpha r)$.

If the plate is clamped on its edge, so $\delta \beta_{r}=0$ at $r=a$, where $a$ is the plate radius, then $J_{1}(\alpha a)=0$, the smallest root for which $J_{1}=0$ is $\alpha a=3.83$. Consequently we 
get the relation for defining critical load

$$
p=1.22 \frac{\left(\phi^{\prime}+\frac{N}{3}\right) h^{2}}{a^{2}} .
$$

Since $\phi^{\prime}=\frac{d \phi}{d s}=E_{t}(s), \quad N=\frac{\sigma_{u}^{0}}{s}=\frac{\phi(s)}{s}=E_{c}(s)$ and $s=\phi^{-1}(p)$, from (4.7) the critical load $p_{c r}$ can be determined.

For elastic stability of plate made of incompressible material $E_{t}(s)=E_{c}(s)=3 G$, the expression (4.7) reduces to the result of Timoshenko [9].

The equation (4.6) may be solved by Bubnov-Galerkin method by putting $\delta \beta_{\text {巾 }}=$ $\operatorname{Ar}(a-r)$, which satisfies conditions $\delta \beta_{r}=0$ at $r=0$ and $r=a$. Substituting the expression of $\delta \beta_{r}$ into (4.6), multiplying obtained result with $r(a-r)$ and integrating over the plate surface, $0 \leq r \leq a, 0 \leq \theta \leq 2 \pi$, gives

$$
p=1.25\left(\phi^{\prime}+\frac{N}{3}\right) \frac{h^{2}}{a^{2}}
$$

the error consists of more than $2 \%$.

\section{Shallow spherical shell}

Points of the middle surface may be referred to coordinates $r$ and $\theta$, the rise of the shell is much smaller than the base radius $a$. We have $R_{2}=R=$ const, $\sin \varphi=\frac{r}{R}$ and approximately $\cos \varphi \approx 1, R_{2} d \varphi=d r$.

The governing equations now have the form

$$
\begin{aligned}
& \frac{\partial}{\partial r}\left(r \delta N_{r}\right)+\frac{\partial}{\partial \theta}\left(\delta N_{r \theta}\right)-\delta N_{\theta}=0 \\
& \frac{\partial}{\partial r}\left(r \delta N_{r \theta}\right)+\frac{\partial \delta N_{\theta}}{\partial \theta}+\delta N_{r \theta}=0 \\
& \frac{\partial^{2}}{\partial r^{2}}\left(r \delta M_{r}\right)+2\left(\frac{\partial^{2} \delta M_{r \theta}}{\partial r \partial \theta}+\frac{1}{r} \frac{\partial \delta M_{r \theta}}{\partial \theta}\right)+\left(\frac{1}{r} \frac{\partial^{2} \delta M_{\theta}}{\partial \theta^{2}}-\frac{\partial \delta M_{\theta}}{\partial r}\right)- \\
& -\frac{r}{R}\left(\delta N_{r}+\delta N_{\theta}\right)-\left[\frac{\partial}{\partial r}\left(r N_{r}^{0} \delta \beta_{r}+r N_{r \theta}^{0} \delta \beta_{\theta}\right)+\frac{\partial}{\partial \theta}\left(N_{r \theta}^{0} \delta \beta_{r}+N_{\theta}^{0} \delta \beta_{\theta}\right)\right]=0
\end{aligned}
$$

where $\delta N_{r}, \delta N_{\theta}, \delta N_{r \theta}$ - generalized force increments

$$
\begin{aligned}
\delta N_{r} & =h\left[\frac{2}{3} N\left(2 \delta \varepsilon_{r}^{*}+\delta \varepsilon_{\theta}^{*}\right)+\left(\phi^{\prime}-N\right) \frac{\sigma_{r}^{0} \delta \varepsilon_{r}^{*}+\sigma_{\theta}^{0} \delta \varepsilon_{\theta}^{*}+2 \sigma_{r \theta}^{0} \delta \varepsilon_{r \theta}^{*}}{\sigma_{u}^{0^{2}}} \sigma_{r}^{0}\right] \\
\delta N_{\theta} & =h\left[\frac{2}{3} N\left(2 \delta \varepsilon_{\theta}^{*}+\delta \varepsilon_{r}^{*}\right)+\left(\phi^{\prime}-N\right) \frac{\sigma_{r}^{0} \delta \varepsilon_{r}^{*}+\sigma_{\theta}^{0} \delta \varepsilon_{\theta}^{*}+2 \sigma_{r \theta}^{0} \delta \varepsilon_{r \theta}^{*}}{\sigma_{u}^{0^{2}}} \sigma_{\theta}^{0}\right], \\
\delta N_{r \theta} & =h\left[\frac{2}{3} N \delta \varepsilon_{r \theta}^{*}+\left(\phi^{\prime}-N\right) \frac{\sigma_{r}^{0} \delta \varepsilon_{r}^{*}+\sigma_{\theta}^{0} \delta \varepsilon_{\theta}^{*}+2 \sigma_{r \theta}^{0} \delta \varepsilon_{r \theta}^{*}}{\sigma_{u}^{0^{2}}} \sigma_{r \theta}^{0}\right]
\end{aligned}
$$


and $\delta M_{r}, \delta M_{\theta}, \delta M_{r \theta}$ - generalized moment increments

$$
\begin{aligned}
\delta M_{r} & =-\frac{h^{3}}{12}\left[\frac{2}{3} N\left(2 \delta \chi_{r}+\delta \chi_{\theta}\right)+\left(\phi^{\prime}-N\right) \frac{\sigma_{r}^{0} \delta \chi_{r}+\sigma_{\theta}^{0} \delta \chi_{\theta}+\sigma_{r \theta}^{0} \delta \chi_{r \theta}}{\sigma_{u}^{0}} \sigma_{r}^{0}\right], \\
\delta M_{\theta} & =-\frac{h^{3}}{12}\left[\frac{2}{3} N\left(2 \delta \chi_{\theta}+\delta \chi_{r}\right)+\left(\phi^{\prime}-N\right) \frac{\sigma_{r}^{0} \delta \chi_{r}+\sigma_{\theta}^{0} \delta \chi_{\theta}+\sigma_{r \theta}^{0} \delta \chi_{r \theta}}{\sigma_{u}^{02}} \sigma_{\theta}^{0}\right], \\
\delta M_{r \theta} & =-\frac{h^{3}}{12}\left[\frac{2}{3} N \delta \chi_{r \theta}+\left(\phi^{\prime}-N\right) \frac{\sigma_{r}^{0} \delta \chi_{r}+\sigma_{\theta}^{0} \delta \chi_{\theta}+\sigma_{r \theta}^{0} \delta \chi_{r \theta}}{\sigma_{u}^{0}} \sigma_{r \theta}^{0}\right],
\end{aligned}
$$

The middle surface kinematic relations in this case are of the form

$$
\begin{aligned}
& \delta \varepsilon_{r}^{*}=\frac{\partial \delta v}{\partial r}+\frac{\delta w}{R}, \quad \delta \varepsilon_{\theta}^{*}=\frac{1}{r}\left(\frac{\partial \delta u}{\partial \theta}+\delta v\right)+\frac{\delta w}{R}, \quad \delta \varepsilon_{r \theta}^{*}=\frac{1}{2}\left[r \frac{\partial}{\partial r}\left(\frac{\delta u}{r}\right)+\frac{1}{r} \frac{\partial \delta v}{\partial \theta}\right] ; \\
& \delta \chi_{r}=\frac{\partial \delta \beta_{r}}{\partial r}, \quad \delta \chi_{\theta}=\frac{1}{r}\left(\frac{\partial \delta \beta_{\theta}}{\partial \theta}+\delta \beta_{r}\right), \quad \delta \chi_{r, \theta}=\frac{1}{2}\left[r \frac{\partial}{\partial r}\left(\frac{\delta \beta_{\theta}}{r}\right)+\frac{1}{r} \frac{\partial \delta \beta_{r}}{\partial \theta}\right], \\
& \delta \beta_{r}=\frac{\partial \delta w}{\partial r}, \quad \delta \beta_{\theta}=\frac{1}{r} \frac{\partial \delta w}{\partial \theta} .
\end{aligned}
$$

The equations $(5.1) \div(5.5)$ lead to a coupled set of three homogeneous equations in $\delta u, \delta v, \delta w$. This set can be reduced to two equations in $\delta w$ and a stress function $F$.

From (5.4) we can get the compatity equation

$$
\frac{1}{r^{2}} \frac{\partial^{2} \delta \varepsilon_{r}^{*}}{\partial \theta^{2}}-\frac{1}{r} \frac{\partial \delta \varepsilon_{r}^{*}}{\partial r}+\frac{1}{r^{2}} \frac{\partial}{\partial r}\left(r^{2} \frac{\partial \delta \varepsilon_{\theta}^{*}}{\partial r}\right)-\frac{1}{r^{2}} \frac{\partial^{2}}{\partial r \partial \theta}\left(r \delta \varepsilon_{r \theta}^{*}\right)=\frac{\Delta \delta w}{R},
$$

where $\Delta=\frac{\partial^{2}}{\partial r^{2}}+\frac{1}{r} \frac{\partial}{\partial r}+\frac{1}{r^{2}} \frac{\partial^{2}}{\partial \theta^{2}}$.

Inversely, from (5.2) the strain increments can be expressed as follows

$$
\begin{aligned}
\delta \varepsilon_{r}^{*}= & \frac{1}{2 N h}\left(2 \delta N_{r}-\delta N_{\theta}\right) \\
& +\frac{1}{4 h}\left(\frac{1}{\phi^{\prime}}-\frac{1}{N}\right)\left[\left(2 \sigma_{r}^{0}-\sigma_{\theta}^{0}\right) \delta N_{r}+\left(2 \sigma_{\theta}^{0}-\sigma_{r}^{0}\right) \delta N_{\theta}+6 \sigma_{r \theta}^{0} \delta N_{r \theta}\right] \frac{2 \sigma_{r}^{0}-\sigma_{\theta}^{0}}{\sigma_{u}^{0^{2}}}, \\
\delta \varepsilon_{\theta}^{*}= & \frac{1}{2 N h}\left(2 \delta N_{\theta}-\delta N_{r}\right) \\
& +\frac{1}{4 h}\left(\frac{1}{\phi^{\prime}}-\frac{1}{N}\right)\left[\left(2 \sigma_{r}^{0}-\sigma_{\theta}^{0}\right) \delta N_{r}+\left(2 \sigma_{\theta}^{0}-\sigma_{r}^{0}\right) \delta N_{\theta}+6 \sigma_{r \theta}^{0} \delta N_{r \theta}\right] \frac{2 \sigma_{\theta}^{0}-\sigma_{r}^{0}}{\sigma_{u}^{0^{2}}}, \\
\delta \varepsilon_{r \theta}^{*}= & \frac{3}{2 N h} \delta N_{r \theta}+\frac{3}{2 h}\left(\frac{1}{\phi^{\prime}}-\frac{1}{N}\right)\left[\left(2 \sigma_{r}^{0}-\sigma_{\theta}^{0}\right) \delta N_{r}+\left(2 \sigma_{\theta}^{0}-\sigma_{r}^{0}\right) \delta N_{\theta}+6 \sigma_{r \theta}^{0} \delta N_{r \theta}\right] \frac{\sigma_{r \theta}^{0}}{\sigma_{u}^{0}},
\end{aligned}
$$

Two first equations (5.1) are satisfied identically if

$$
\delta N_{r}=\frac{1}{r} \frac{\partial F}{\partial r}+\frac{1}{r^{2}} \frac{\partial^{2} F}{\partial \theta^{2}}, \quad \delta N_{\theta}=\frac{\partial^{2} F}{\partial r^{2}}, \quad \delta N_{r \theta}=-\frac{\partial}{\partial r}\left(\frac{1}{r} \frac{\partial F}{\partial \theta}\right)
$$


The third equation of (5.1)and the equation (5.6) in use of expressions (5.3), (5.7) and (5.8) lead to the equations for $w$ and $F$.

For example we consider a spherical cap subjected to uniform external pressure $p$. Let us assume the prebuckling state may be approximated by a membrane analysis, then $N_{r}^{0}=N_{\theta}^{0}=-p \frac{R}{2}, N_{r \theta}^{0}=0$, so that $\sigma_{r}^{0}=\sigma_{\theta}^{0}=-\frac{p R}{2 h}, \sigma_{r \theta}^{0}=0, \sigma_{u}^{0}=\left|\sigma_{r}^{0}\right|=\frac{p R}{2 h}$, and $\sigma_{u}^{0}=\phi(s)$.

In this case, the third equation of (5.1) has the form

$$
\begin{aligned}
\frac{\partial^{2}}{\partial r^{2}}\left(r \delta M_{r}\right) & +2\left(\frac{\partial^{2} \delta M_{r \theta}}{\partial r \partial \theta}+\frac{1}{r} \frac{\partial \delta M_{r \theta}}{\partial \theta}\right) \\
& +\frac{1}{r} \frac{\partial^{2} \delta M_{\theta}}{\partial \theta^{2}}-\frac{\partial \delta M_{\theta}}{\partial r}-\frac{r}{R}\left(\delta N_{r}+\delta N_{\theta}\right)-\frac{p R}{2} \Delta w=0
\end{aligned}
$$

and the internal moment increments (5.3), the strain increments (5.7) are rewritten as follows

$$
\begin{aligned}
\delta M_{r} & =-\frac{h^{3}}{12}\left[\frac{2 N}{3}\left(2 \frac{\partial^{2} \delta w}{\partial r^{2}}+\frac{1}{r^{2}} \frac{\partial^{2} \delta w}{\partial \theta^{2}}+\frac{1}{r} \frac{\partial \delta w}{\partial r}\right)+\left(\phi^{\prime}-N\right) \Delta \delta w\right] \\
\delta M_{\theta} & =-\frac{h^{3}}{12}\left[\frac{2 N}{3}\left(\frac{2}{r^{2}} \frac{\partial^{2} \delta w}{\partial \theta^{2}}+\frac{2}{r} \frac{\partial \delta w}{\partial r}+\frac{\partial^{2} \delta w}{\partial r^{2}}\right)+\left(\phi^{\prime}-N\right) \Delta \delta w\right] \\
\delta M_{r \theta} & =-\frac{h^{3}}{18} N\left(\frac{1}{r} \frac{\partial^{2} \delta w}{\partial r \partial \theta}-\frac{1}{r^{2}} \frac{\partial \delta w}{\partial \theta}\right) . \\
\delta \varepsilon_{r}^{*} & =\frac{1}{2 N h}\left(\frac{2}{r} \frac{\partial F}{\partial r}+\frac{2}{r^{2}} \frac{\partial^{2} F}{\partial \theta^{2}}-\frac{\partial^{2} F}{\partial r^{2}}\right)+\frac{1}{2 h}\left(\frac{1}{\phi^{\prime}}-\frac{1}{N}\right) \Delta F \\
\delta \varepsilon_{\theta}^{*} & =\frac{1}{2 N h}\left(2 \frac{\partial^{2} F}{\partial r^{2}}-\frac{1}{r} \frac{\partial F}{\partial r}-\frac{1}{r^{2}} \frac{\partial^{2} F}{\partial \theta^{2}}\right)+\frac{1}{2 h}\left(\frac{1}{\phi^{\prime}}-\frac{1}{N}\right) \Delta F \\
\delta \varepsilon_{r \theta}^{*} & =\frac{3}{2 N h}\left(\frac{1}{r^{2}} \frac{\partial F}{\partial \theta}-\frac{1}{r} \frac{\partial^{2} F}{\partial r \partial \theta}\right) .
\end{aligned}
$$

Introduction of expressions (5.8), (5.10) into equation (5.9) and expressions (5.11) into equation (5.6) gives

$$
\begin{aligned}
& \frac{h^{3}}{12}\left(\frac{N}{3}+\phi^{\prime}\right) \Delta \Delta \delta w+\frac{p R}{2} \Delta \delta w=-\frac{\Delta F}{R}, \\
& \left(\frac{1}{N}+\frac{1}{\phi^{\prime}}\right) \Delta \Delta F=\frac{2 h}{R} \Delta \delta w .
\end{aligned}
$$

The Bubnov-Galerkin method can be applied to the system of equations (5.12), (5.13) by choosing expressions of $F$ and $\delta w$, satisfying boundary conditions. In results we get the equation for finding critical load. Otherwise, because of the appearance of only Laplace operator in (5.12), (5.13) we can use the following coordinate transformation [7]

$$
x=r \cos \theta, \quad y=r \sin \theta, \quad \Delta=\frac{\partial^{2}}{\partial r^{2}}+\frac{1}{r} \frac{\partial}{\partial r}+\frac{1}{r^{2}} \frac{\partial r^{2}}{\partial \theta^{2}}=\frac{\partial^{2}}{\partial x^{2}}+\frac{\partial^{2}}{\partial y^{2}},
$$


the differential equations are seen to be satisfied by solutions of the form

$$
\delta w=\sin \left(\frac{k_{1} x}{R}\right) \sin \left(\frac{k_{2} y}{R}\right), \quad F=C \sin \left(\frac{k_{1} x}{R}\right) \sin \left(\frac{k_{2} y}{R}\right),
$$

where $k_{1}, k_{2}$ are wavelength parameters. Introduction of these expressions into

$$
\text { gives } C=-\frac{2 h R}{\left(\frac{1}{N}+\frac{1}{\phi^{\prime}}\right)\left(k_{1}^{2}+k_{2}^{2}\right)} \cdot \text { Substituting obtained result for } F \text { and }
$$

expression of $\delta w$ into (5.12) yields

$$
p=\frac{4 h}{R\left(\frac{1}{N}+\frac{1}{\phi^{\prime}}\right)}\left[\frac{1}{k_{1}^{2}+k_{2}^{2}}+\frac{h^{2}}{24 R^{2}}\left(\frac{N}{3}+\phi^{\prime}\right)\left(\frac{1}{N}+\frac{1}{\phi^{\prime}}\right)\left(k_{1}^{2}+k_{2}^{2}\right)\right] .
$$

An approximate expression for the critical pressure may be obtained by minimization of $p$ with respect to $k_{1}^{2}+k_{2}^{2}$. The smallest $p$ is found as following

$$
p=\frac{4}{3 \sqrt{2}}\left(\frac{h}{R}\right)^{2} \sqrt{\frac{\left(N+3 \phi^{\prime}\right) N \phi^{\prime}}{N+\phi^{\prime}}} .
$$

Taking into account $N=\frac{\sigma_{u}^{0}}{s}=\frac{\phi(s)}{s}=E_{c}(s), \phi^{\prime}=E_{t}(s), s=\phi^{-1}\left(\sigma_{u}^{0}\right)$; by the loading parameter method from (5.14) we can get the critical pressure $p_{c r}$.

For an elastic shell of incompressible material $E_{c}(s)=E_{t}(s)=3 G$, from (5.14) we obtain

$$
p_{c r}=4 G\left(\frac{h}{R}\right)^{2}
$$

this value is the same as that given in $[2,7]$.

Remark. In [8] these two particular cases; circular plate under uniform compressive load and spherical cap under external pressure, have been considered by use of the incremental theory of plasticity and the deformation theory of plasticity by generalizing directly formulae of elastic solutions. But our formulation can be applied not only to these particular cases, but to more general cases of shells of revolution as well. The investigated cases only play a role of illustration of the method.

\section{Conclusions}

The governing equations of the elastoplastic stability problem of shells of revolution subjected to complex loading are derived by using theory of elastoplastic processes and the adjacent-equilibrium criterion.

The Bubnov-Galerkin method and the loading parameter method can be used for solving problem, in some particular investigated cases we can get analytical solutions. 
The elasto-plastic stability of circular plates and spherical shells is investigated. Obtained expressions of critical loads reduce to results of Timoshenko and Hutchinson for elastic shells.

This publication is completed with financial support of the Council for Natural Science of Vietnam.

\section{REFERENCES}

1. Brush D. O., Almroth B. O. Buckling of bars, plates and shells. Mc Graw-Hill 1975.

2. Bushnell D. Stress, stability and vibration of complex branched shells of revolution. Proc. AIAA/ASME/SAE 14th Struct, Dyn. Mater. Conf. Williamsburg Va, 1973.

3. Dao H. B. Theory of Elasticity. VNU Publishing House 2000.

4. Dao H. B. Theory of Elastoplastic processes. VNU Publishing House 1999.

5. Grigolyuk E. I. Loss of stability of thin plastic shells without unloading. Prikl. Math. Mech. 21, 1957, 846-849.

6. Hill R. Plastic deformation and instability in thin-walled tubes under combined loading: a general theory. Journal of. Mech. and Phys. of Solids 47, 1999, 921-933.

7. Hutchinson J. W. Imperfection sensitivity of externally pressurized spherical shells. Journal of Appl. Mech. 34, 1967, 49-55.

8. Lubliner J. Plasticity theory. Macmillan Publishing Company 1990.

9. Timoshenko S. P., Gere J. M. Theory of elastic stability, 2d ed. Mc Graw-Hill 1961.

10. Ülo Lepik. Bifurcation analysis of elastic-plastic cylindrical shells. Int. Journal of Non-linear Mech. 34, 1999, 299-311.

Received October 4, 2002

VỀ BÀI TOÁN ỔN ĐỊNH ĐÀN DÉO CỦA VỎ TRÒN XOAY

Bài toán ổn định đàn hồi của vỏ tròn xoay đã được giải quyết, tuy nhiên ổn định đàn déo còn ít được quan tâm. Trong bài báo này, sử dụng lý thuyết quá trình đàn dẻo tác giả thiết lập các hệ thức cơ bản của bài toán ổn định đàn dèo của vó tròn xoay chịu quá trình đặt tải phức tạp. Có thể sứ dụng phương pháp Bubnov-Galerkin và phương pháp tham số tải để giải bài toán, trong một số trường hợp riêng có thể nhận được nghiệm giải tích. Để minh họa đã khảo sát ổn định đàn déo của bản tròn và vó cầu. Từ biểu thức nhận được của lực tới hạn có thể nhận lại kết quá của Timoshenko và Hutchinson cho vỏ đàn hồi. Điều này bảo đảm độ tin cậy của phương pháp tính toán. 